\title{
Detecting Majorana fermions by use of superconductor-quantum Hall liquid junctions
}

\author{
Zheng-Wei Zuo ${ }^{1}$, Huijuan Li ${ }^{1}$, Liben Li ${ }^{1}$, L. Sheng ${ }^{2}$, R. Shen ${ }^{2}$ and D. Y. Xing ${ }^{2}$ \\ 1 School of Physics and Engineering, Henan University of Science and Technology, Luoyang 471003, China \\ 2 National Laboratory of Solid State Microstructures, Department of Physics, and Collaborative Innovation Center of \\ Advanced Microstructures, Nanjing University, Nanjing 210093, China
}

PACS 73.43.-f - Quantum Hall effects

PACS 74.45.+c - Proximity effects; Andreev reflection; SN and SNS junctions

PACS 64.60.ae - Renormalization-group theory

\begin{abstract}
The point contact tunnel junctions between a one-dimensional topological superconductor and single-channel quantum Hall $(\mathrm{QH})$ liquids are investigated theoretically with bosonization technology and renormalization group methods. For the $\nu=1$ integer QH liquid, the universal low-energy tunneling transport is governed by the perfect Andreev reflection fixed point with quantized zero-bias conductance $G(0)=2 e^{2} / h$, which can serve as a definitive fingerprint of the existence of a Majorana fermion. For the $\nu=1 / m$ Laughlin fractional QH liquids, its transport is governed by the perfect normal reflection fixed point with vanishing zero-bias conductance and bias-dependent conductance $G(V) \sim V^{m-2}$. Our setup is within reach of the present experimental techniques.
\end{abstract}

Introduction. - Due to their potential applications in fault-tolerant topological quantum computation [1], Majorana fermions have been of great interest in condensed matter physics over the past decade [2-4]. Several solid-state candidates for experimentally realizing topological superconductors (TSC) with the Majorana fermions have been put forward, based on proximity coupling to $s$-wave superconductors, such as the topological insulators $[5,6]$, semiconductor quantum wires with spin-orbit interaction [7-9], and quasi one-dimensional (1D) chains of magnetic atoms [10-14].

At present, verifying and detecting the existence of Majorana fermions remains an outstanding experimental challenge [4]. The possible experimental evidences of Majorana fermions have been shown in spin-orbit coupled quantum wires subjected to a magnetic field and proximate to an $s$-wave superconductor [15-17]. A zerobias conductance peak (ZBCP) was reported by these experiments. However, other explanations of this anomaly ZBCP were also suggested [18-20]. How to distinguish the ZBCP caused by the Majorana fermion from those by other mechanisms is an important task. There are other proposals for detecting the Majorana fermions in TSC [21-25].

For a TSC coupled to other materials via point con- tact, the presence of Majorana fermions can lead to many interesting transport properties [26-36]. When a normal metal lead, either noninteracting or interacting, couples to Majorana fermions through electron tunneling, the Majorana fermions can induce perfect Andreev reflections and may result in a quantized zero-bias conductance $2 e^{2} / h$ at low energies [26,27]. For the normal metal lead, various parameters such as the electronic interaction, disorder, and finite length need to be taken into account [27-36], and they can result in a drastic departure from the noninteracting cases. These factors have been analyzed by scattering matrix theory [26], Keldysh formalism [34], the Luttinger liquids theory and renormalization group method $[37,38]$. It is highly desirable to overcome effects of these factors on the tunneling spectrum. If the normal metal lead is replaced with a quantum Hall (QH) liquid [39-41], the situation will be quite different. The QH liquid is a topologically nontrivial system with topologically-protected edge states. For the single-channel QH liquid lead, the electronic interactions are suppressed or fixed, and effects of disorder and finite length are also removed. No study to date, however, has examined the advantages of using the $\mathrm{QH}$ liquid-TSC tunnel junctions. The integer (fractional) QH states show precisely quantized plateaus at integer (fractional) Hall conductance val- 
ues of $e^{2} / h$. The effects of electronic interactions are suppressed in the integer QH liquids. There are fractionallycharged quasiparticles with fractional exchange statistics in fractional QH states $[42,43]$. The integer and fractional $\mathrm{QH}$ states support gapless edge excitations. Due to these intriguing and exotic properties of the QH liquids, it is of both theoretical and practical interest to investigate transport properties of the junctions between the TSC and QH liquids.

In this work, we investigate the point contact tunnel junctions between a 1D TSC and single-channel QH liquids with bosonization technology and renormalization group methods. For the $\nu=1$ integer $\mathrm{QH}$ liquid, the perfect Andreev reflection with quantized zero-bias conductance $2 e^{2} / h$ at zero temperature is predicted to occur, which is caused by the Majorana fermion induced tunneling rather than by the conventional Cooper-pair tunneling. Such a quantized conductance can serve as a definitive fingerprint of a Majorana fermion, which is robust against details of the setup. On the other hand, for the Laughlin fractional $\mathrm{QH}$ liquids $(\nu=1 / m$ with $m$ the odd integral greater than 1), the universal low-energy transport is governed by the fixed point describing perfect normal reflection, which leads to a vanishing zero-bias conductance and bias-dependent conductance of $G(V) \sim V^{m-2}$. If the $\mathrm{SC}$ is topologically trivial, the bias-dependent conductance becomes $G(V) \sim V^{4 m-2}$. From these behaviors of $G(V)$, we can extract the topological invariant $m$ controlled by the bulk fractional QH states.

Theory and Discussion. - The point contact tunnel junction we study is formed by a single-channel QH liquid adjacent to a 1D TSC, as depicted in figure 1 . The 1D TSC can be obtained by a spin-orbit coupled quantum wire subjected to a magnetic field and proximate to an $s$ wave superconductor $[7,8]$. The $1 \mathrm{D}$ TSC is characterized by the localized Majorana modes $\gamma$ and $\gamma^{\prime}$ at end points. It is assumed that all the important energy scales are smaller than the superconducting energy gap that is taken as the unit of energy.

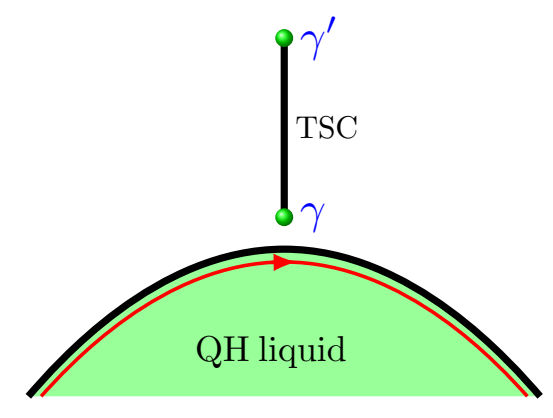

Fig. 1: (Color online) Schematic illustration of the tunnel junction between a 1D TSC and a single-channel QH liquid. The TSC is characterized by Majorana modes $\gamma$ and $\gamma^{\prime}$. The edge of the $\mathrm{QH}$ state can be described in terms of chiral bosonic field $\phi$.
There are two types of single-channel $\mathrm{QH}$ liquid with filling fraction $\nu=1 / m$ where $m$ is an odd integer. One is the integer $\mathrm{QH}$ liquid with $\nu=m=1$ and the other is the Laughlin fractional $\mathrm{QH}$ liquid with $m \geq 3$. Because of the high magnetic field, the electrons in the QH liquids are fully spin-polarized (spin up). The model Hamiltonian for the whole system can be written as

$$
H=H_{0}+H_{T},
$$

where $H_{0}$ is the edge Hamiltonian of the QH liquid and $H_{T}$ is the tunneling Hamiltonian at the point contact. For the single-channel QH liquid, the edge excitations are described by a single-channel chiral Fermi (or Luttinger) liquid $[43,44]$. The edge Hamiltonian of QH liquid is given by

$$
H_{0}=\frac{m v_{F}}{4 \pi} \int d x\left(\partial_{x} \phi(x)\right)^{2},
$$

with $\phi(x)$ a chiral boson field and $v_{F}$ the Fermi velocity. The electron density operator is given by $\rho(x)=$ $\partial_{x} \phi(x) / 2 \pi$. The electron operators can be expressed as

$$
\Psi_{\alpha}(x)=\Gamma_{\alpha} e^{i m \phi(x)},
$$

with $\Gamma_{\alpha}$ the Klein factors which obey $\Gamma_{\alpha}^{\dagger}=\Gamma_{\alpha}$ and $\left\{\Gamma_{\alpha}, \Gamma_{\beta}\right\}=2 \delta_{\alpha \beta}$ to ensure the anti-commutation relations between different fermion species. The Klein factors can be viewed as additional Majorana fermions, which is important for studying related Majorana fermion models $[45,46]$. Because of the anti-commutation relations of electrons $\Psi_{\alpha}$ and Majorana fermion $\gamma$, we require the relation $\left\{\Gamma_{\alpha}, \gamma\right\}=0$.

Now we consider the tunneling at the point contact. In general, there are two major types of tunneling processes: the Majorana fermion-induced tunneling and the regular Cooper pairs tunneling. First, we consider the process of Majorana fermion $\gamma$ coupled to electrons of the $\mathrm{QH}$ liquid $\gamma\left[\Psi(0)-\Psi^{\dagger}(0)\right]$. We assume the 1D TSC is sufficiently long so that Majorana fermion $\gamma^{\prime}$ does not couple to electrons in the QH liquid. Since the electrons in the QH liquid are fully spin-polarized (or effectively spinless), they can couple to the Majorana mode $\gamma$ and undergo the selective equal-spin Andreev reflection [47], which is in analogy to the perfect Andreev reflection in the TSC/helical Luttinger liquid junction $[27,28]$.

Second, whether the superconducting wire is in a topologically nontrivial or trivial phase, the Cooper-pair tunneling may occur. The regular Cooper pairs may hop from the superconducting wire into the $\mathrm{QH}$ liquid via the point contact, which is induced locally in the wire by the superconducting pairing $[27,32,33,48]$, similarly to the regular Cooper-pair tunneling in superconductor-Luttinger liquid proximity systems [49-53]. Since the edge states in the QH liquid can only transport spin-up electrons, the regular spin singlet Cooper-pair tunneling process needs to complete two processes [54]. The first one is that the Cooper pair breaks apart into two unpaired electrons, as in the 
conventional Andreev process, where the quantum fluctuations of superconducting phase have been neglected. The second one is the spin-flip process to align the spin of both electrons, which can be mediated by spin-orbit coupling in the $\mathrm{QH}$ liquid. The two processes can be synthesized as $\Psi_{\uparrow}^{\dagger}(0) \Psi_{\uparrow}^{\dagger}(\xi)+H . c$, with $\xi$ the superconducting coherence length. If the spin-up subscript is dropped, the tunneling Hamiltonian of Cooper pairs can be written as $\Psi^{\dagger} \Psi^{\dagger}+$ H.c. Thus, the tunneling Hamiltonian defined at $x=0$ (the right and left sides of the junction are labelled by $x>0$ and $x<0$ respectively) reads

$$
H_{T}=t \gamma\left[\Psi(0)-\Psi^{\dagger}(0)\right]+\Delta\left[\Psi^{\dagger} \Psi^{\dagger}+H . c\right],
$$

where the first term describes the Majorana fermion tunneling with $t$ coupling amplitude and the second term accounts for regular Cooper-pair tunneling with $\Delta$ coupling amplitude.

According to the bosonization technology [37,38], the tunneling Hamiltonian can be expressed as

$$
H_{T}=2 i t \gamma \Gamma \cos [m \phi(0)]+2 \Delta \cos 2 m \phi(0),
$$

where the first term factorizes into the Klein-Majorana interaction and charge sector parts. The Majorana fermion enters the problem only via $\gamma \Gamma$. The ordinary fermion can be defined as $\psi=(\gamma+i \Gamma) / 2$ with $\left\{\psi, \psi^{\dagger}\right\}=1$, so that we have

$$
i \gamma \Gamma=2 \psi^{\dagger} \psi-1= \pm 1,
$$

where \pm correspond to the energy level being occupied and empty, respectively. Consequently, the Klein-Majorana fusion procedure can eliminate the Majorana degrees of freedom $[45,46]$ and simplify our theoretical calculations.

In what follows, we use perturbative renormalization group (RG) analysis to uncover the transport signature of the tunneling junction. As the first step, we integrate out the chiral bosonic field $\phi$ in the imaginary-time partition function of the Hamiltonian in equation (1) except at $x=$ 0 . The partition function can be written as a path integral

$$
\mathcal{Z}=\int \mathcal{D} \phi e^{-\left(S_{0}+S_{T}\right)},
$$

where the effective actions are given by

$$
S_{0}=\frac{m}{2 \pi} \int \frac{d \omega}{2 \pi}|\omega||\phi(\omega)|^{2},
$$

and

$$
S_{T}=\int d \tau[2 t \cos [m \phi(\tau)]+2 \Delta \cos 2 m \phi(\tau)] .
$$

Next, we perform weak-coupling RG analysis in the frequency domain. The bosonic field $\phi$ is separated into slow $(s)$ and fast $(f)$ modes: $\phi_{s}(\tau)=\int_{-\Lambda / b}^{\Lambda / b} \frac{d \omega}{2 \pi} e^{-i \omega \tau} \phi(\omega)$ and $\phi_{f}(\tau)=\int_{\Lambda / b<|\omega|<\Lambda} \frac{d \omega}{2 \pi} e^{-i \omega \tau} \phi(\omega)$, with $\Lambda$ as an energy cutoff, $b>1$ as a scale factor, and $\tau=i t$ the Euclidean time.
After integrating over the fast modes, the new effective action can be calculated using cumulant expansion to the lowest-order approximation in coupling $t$ and $\Delta$, yielding

$$
e^{-S_{e f f}\left[\phi_{s}\right]} \approx e^{-S_{s}\left[\phi_{s}\right]} e^{-\left\langle S_{T}\left[\phi_{s}, \phi_{f}\right]\right\rangle_{f}},
$$

where $\langle\ldots\rangle_{f}$ denotes integrating out the fast mode, and $S_{s}\left[\phi_{s}\right]$ is the slowly fluctuating part of $S$. The lowestorder flows of the coupling $t$ and $\Delta$ under the RG are given by

$$
\begin{aligned}
\frac{d t}{d \ln b} & =t\left(1-\frac{m}{2}\right), \\
\frac{d \Delta}{d \ln b} & =\Delta(1-2 m),
\end{aligned}
$$

with $\ln b$ as the dimensionless flow parameter. For the $p$-wave Cooper-pair tunneling, we have $\Delta\left(\Psi^{\dagger} \partial_{x} \Psi^{\dagger}+H . c\right) \propto \sin 2 m \phi$, from which the same lowest-order flow equation as equation (12) can be obtained. In addition, we may in principle add other types of coupling at the point contact, including even higher-order derivatives in $\Psi^{\dagger}(0)$ and/or $\Psi(0)$, but it can be shown that they are generally irrelevant $[27,28]$.

From these flow equations (11) and (12), we can reach the following conclusions. For all single-channel QH liquid, the Cooper-pair tunneling is irrelevant. For the integer QH liquid of $m=1$, the tunneling process of Majorana fermions coupling to electrons of the QH liquid is relevant. However, for the Laughlin fractional QH liquid of $m \geq 3$, the Majorana fermions tunneling is irrelevant. So, for $1 \mathrm{D}$ TSC/integer $\mathrm{QH}$ liquid tunnel junction, the perfect normal reflection fixed point is unstable toward the perfect Andreev reflection fixed point, which is analogues to the hybrid system of superconductor trench and QH liquid [30]. In what follows, we first analyze the stability of the perfect Andreev reflection fixed point and then the transport properties of $1 \mathrm{D} \mathrm{TSC} /$ fractional $\mathrm{QH}$ liquid tunnel junction.

Integer QH liquid. In the case of $m=1$, one sees that the tunneling term $t$ is relevant. The universal low-energy transport is governed by the fixed point describing perfect Andreev reflection with quantized zero-bias conductance $G(0)=2 e^{2} / h$. At this fixed point, the nontrivial boundary condition $\Psi^{\dagger}\left(0^{+}\right)=e^{i \alpha} \Psi\left(0^{-}\right)\left(0^{ \pm}\right.$stands for the right and left sides of the point contact junction) needs to be satisfied $[27,30]$. Here, we set $\alpha=0$ for simplicity and this boundary condition implies $\phi\left(0^{+}\right)=-\phi\left(0^{-}\right)$. Next, we investigate the stability of the perfect Andreev reflection fixed point. Physically, the deviations from this fixed point mean that the electrons $\psi(x)=e^{i \phi(x)}$ (we have neglected the Klein factor. For the fractional QH liquid, the $e^{i \phi(x)}$ is fractionally-charged quasiparticle) transmission between left and right edge parts of $\mathrm{QH}$ liquid at $x=0$ is allowed. So, the leading perturbation away from the perfect Andreev reflection fixed point is the electrons (fractionally-charged quasiparticles for the fractional $\mathrm{QH}$ liquid) transmission along the edge of the $\mathrm{QH}$ liquid at 
$x=0$

$$
\delta S_{u}=u \int d \tau\left[\psi^{\dagger}\left(0^{+}\right) \psi\left(0^{-}\right)+H . c\right] .
$$

According to the RG transformation, the lowest-order flow of coupling $u$ can be expressed as

$$
\frac{d u}{d \ln b}=u\left(1-\frac{2}{m}\right)
$$

from which, it follows that for the integer $\mathrm{QH}$ liquid $(m=1)$, the electrons transmission is irrelevant. As a result, the perfect Andreev reflection fixed point due to the Majorana mode coupling is stable and the resulting ZBCP in tunneling spectra is robust. For the fractional $\mathrm{QH}$ liquid, the quasiparticles transmission is relevant and the perfect Andreev reflection fixed point is unstable.

Based on the analysis above, we can conclude that for the 1D TSC/integer QH liquid tunnel junction, there is a perfect Andreev reflection fixed point with zero-bias tunneling conductance $G(0)=2 e^{2} / h$ at zero temperature and voltage. Such a quantized zero-bias tunneling conductance is robustly independent of those irrelevant parameters, so long as the coupling of the Majorana modes to the integral QH liquid remains finite. In contrast, for the topologically trivial superconductor ( $s$-wave or $p$-wave) in the absence of a Majorana fermion, the ground state corresponds to a perfect normal reflection fixed point with a vanishing zero-bias tunneling conductance $G(0)=0$. From the distinguishing difference in zero-bias tunneling conductance between the superconductors in the presence and absence of Majorana modes, it follows that the quantized conductance $G(0)=2 e^{2} / h$ can serve as a definitive fingerprint of a Majorana fermion at the 1D TSC/integer QH liquid tunnel junction, and the $\mathrm{ZBCP}$ can be regarded as an illustrative example of Majorana fermion-induced equalspin Andreev reflections. Compared to the normal metal lead [26-29], there are outstanding advantages of integer $\mathrm{QH}$ liquid lead. The electronic interactions in the integer $\mathrm{QH}$ liquid are suppressed. The disorder plays a decisive role in the formation of the observed integer $\mathrm{QH}$ plateaus [55]. So, the effects of various factors such as electronic interactions, disorder, and finite length on the tunneling spectrum are removed.

Fractional QH liquids. From the equations (11) and (12), we can see that for the Laughlin fractional QH liquid, the Majorana fermions and Cooper-pair tunneling are irrelevant. The perfect normal reflection fixed point is stable. From equation (14), it is found that the quasiparticles transmission is relevant and the perfect Andreev reflection fixed point is unstable. So, no matter that the superconducting wire is nontrivial or trivial, the low-energy physics for all Laughlin fractional QH liquids is governed by the perfect normal reflection fixed point with a vanishing zerobias conductance, $G(0)=0$.

Based on the analysis above, when the superconducting wire is nontrivial, the leading-order perturbation around the perfect normal reflection fixed point is the Majorana fermion-induced tunneling. To make a connection with experiments, it is important to take into account the corrections to the tunneling conductance due to finite bias voltage or temperature. According to the equation (11), we can calculate the tunneling conductance to the lowestorder approximation in bias voltage $V$ or temperature $T$, yielding

$$
G(V) \sim V^{m-2}, G(T) \sim T^{m-2} .
$$

This power law vanishing form can be understood by the following argument. First, the quasiparticles in the Laughlin fractional $\mathrm{QH}$ liquid are anyons with fractional charge and fractional exchange statistics, which are not allowed for tunneling from the Laughlin fractional QH liquid to the TSC. These anyons must form into electrons for tunneling. Second, the Majorana fermion (Majorana bound state) is the Bogoliubov quasiparticle with equal superposition of a electron and a hole. On the other hand, fractional QH edge states are topological many-body systems with strong repulsive interaction, which make the Majorana fermions tunneling strongly inhibited in contrast to integer $\mathrm{QH}$ liquid case. Third, the fractional $\mathrm{QH}$ liquid is phase coherence state and does not dissipate, so the Majorana fermions of TSC do not reach equilibrium with the reservoir of edge excitations of the fractional $\mathrm{QH}$ liquid. In other words, the physics of Majorana fermioninduced tunneling is determined by the physical properties of quasiparticles in the fractional QH liquid and Majorana fermions in TSC. All these factors make the point contact insulating.

For the trivial superconducting wire, the leading-order perturbation around the perfect normal reflection fixed point is the regular Cooper-pair tunneling due to absence of Majorana fermions. So, for a Laughlin QH liquid coupled to a topologically trivial $s$-wave ( $p$-wave) superconductor, the tunneling conductance is given by [54]

$$
G(V) \sim V^{4 m-2}, G(T) \sim T^{4 m-2},
$$

which is also applicable to the integral QH liquid case. As discussed above, the regular Copper-pair tunneling is composite process. The Cooper pair first breaks into two unpaired electrons. Then, the spin-down electron is changed into a spin-up electron by the spin-flip process. Because of the Pauli exclusion principle, these two spin-up electrons can not occupy the same state (position). One electron needs to get away some certain distance for the other to transfer. For the $p$-wave Cooper-pair tunneling, there is no spin-flip process but the Pauli exclusion principle still plays a role on the tunneling. On the basis of the strongly repulsive Laughlin fractional $\mathrm{QH}$ liquid, the tunneling of the $s$-wave and $p$-wave Cooper pairs is strongly inhibited at low energies because of the Coulomb blockade effect and Pauli exclusion principle.

A comparison between equations (15) and (16) indicates that although the zero-bias tunneling conductances are 
vanishing in both cases, they exhibit quite different powerlaw scaling with both bias and temperature for fixed $m$, from which one can judge whether the Majorana fermion is present or absent in the superconducting wire. Take the $\nu=1 / 3$ Laughlin fractional QH liquid as example. For the 1D TSC, the tunneling conductance varies linearly with bias voltage or temperature at low energies, while for the conventional superconductor, it is proportional to $V^{10}$ (or $\left.T^{10}\right)$. From the experimental observation of tunneling conductance, it is easy to distinguish its linear scaling with $V$ from the tenth power of $V$, providing an indication of the existence of the Majorana modes in the 1D TSC. On the other hand, from the tunneling conductance behavior, we can extract the topological invariant $m$ controlled by the bulk fractional QH states, whose value does not depend on detailed properties of edges. Similar to the integer QH liquid case, the electronic interactions in the fractional $\mathrm{QH}$ liquid are fixed. The fractional $\mathrm{QH}$ states are robust to impurity such as disorder. Thus, the TSC/fractional QH liquid tunnel junction has the same outstanding advantages of the integer $\mathrm{QH}$ liquid case.

The transport measurements of our setup are within reach of present experimental techniques. The 1D spinorbit coupled wire can be realized using an InSb nanowire with effective mass $m^{*}=0.015 m_{e}$ [15]. The $g$ factor is equal to $g=50$, the magnetic field $B \sim 0.15 T$, and the Rashba spin-orbit coupled $E_{S O} \sim 50 \mu \mathrm{eV}$. Its proximity to superconducting $\mathrm{NbTiN}$ electrodes can induce a superconducting gap of about $250 \mu \mathrm{eV}$. On the other hand, the $\mathrm{QH}$ liquid can be obtained by GaAs-AlGaAs heterojunctions [40]. However, the QH liquids need high magnetic field, which destroys the superconductivity of the s-wave superconductor in TSC. We can use a type-II s-wave superconductor in TSC compatible with the high magnetic field in $\mathrm{QH}$ liquids. In addition, the $\nu=1$ integer $\mathrm{QH}$ liquid can be achieved by the quantum anomalous Hall states in magnetic topological insulators [56], which do not need the high magnetic field. Furthermore, the quantum point contacts have been used widely as transport measurements in solid-state systems.

Table 1: The tunneling conductances at finite voltage and zero temperature of four tunnel junction archetypes. The columns denote two types of single-channel $\mathrm{QH}$ liquids, and the rows indicate the superconductors (SC) with and without Majorana zero-mode bound states.

\begin{tabular}{lll} 
& integral QH & fractional QH \\
\hline topological SC & $\frac{2 e^{2}}{h}\left(1-c_{V} V^{2}\right)^{*}$ & $\sim V^{m-2}$ \\
conventional SC & $\sim V^{2}$ & $\sim V^{4 m-2}$ \\
\hline
\end{tabular}

$c_{V}$ denotes a non-universal constant

Conclusions. - The point contact tunnel junctions between a 1D TSC and single-channel QH liquids have been investigated by bosonization technology and renormalization group methods. The main results are partially reported in table 1 . Due to different correlated and topo- logical properties, the tunneling conductance behaviors of four types of junctions are quite different from each other. For a 1D TSC coupled to a $\nu=1$ integer $\mathrm{QH}$ liquid, the low-energy physics is governed by the perfect Andreev reflection fixed point. We have proposed an experimentally accessible scheme with relatively simple geometry to detect the ZBCP, the tunneling being induced by the Majorana modes rather than by the conventional Cooper pairs. As a result, the quantized zero-bias conductance $2 e^{2} / h$ can serve as a definitive fingerprint of the existence of Majorana fermions. If the Majorana fermion is absence, we have $G(V) \sim V^{2}$, exhibiting a vanishing zero-bias conductance. In the $\nu=1 / m$ Laughlin $\mathrm{QH}$ liquid case, the universal low-energy transport is governed by the fixed point describing perfect normal reflection, and the bias-dependent tunneling conductance is given by $G(V) \sim V^{m-2}$ and $V^{4 m-2}$, respectively, for the 1D TSC and conventional SC. The temperature and voltage dependence of the tunneling conductance reflect the topological structure of the fractional QH liquids. From the behavior of tunneling conductance, we can extract the topological invariant $m$ controlled by the bulk fractional $\mathrm{QH}$ states. An outstanding advantage of using the $\mathrm{QH}$ liquids is that only two factors, the topological character of the TSC and filling $\nu$ of the $\mathrm{QH}$ liquid, determine the characteristic of tunneling conductance at low energy, the others such as electronic interaction strength, disorder, and finite length of the lead are irrelevant. There are some open works for future research. We can use the BTK theory [57] with scaling approach and entire S-matrix [53] to recalculate the tunneling transport properties and discuss the effects of geometry, boundaries, multi-band, and disorder of the superconducting wire and incoherent reservoir. A numerical study of our system would be very useful, checking our predicted phase diagram.

$$
* * *
$$

This work was supported by the National Natural Science Foundation of China under grant numbers 11447008 (Z.Z.W.), 11225420 (L.S.), and 11474149 (R.S.), the State Key Program for Basic Researches of China under grants numbers 2014CB921103 (L.S.) and 2011CB922103 (D.Y.X.), and a project funded by the PAPD of Jiangsu Higher Education Institutions.

\section{REFERENCES}

[1] Nayak C., Simon S. H., Stern A., Freedman M. and Das Sarma S., Rev. Mod. Phys., 80 (2008) 1083.

[2] Alicea J., Rep. Prog. Phys., 75 (2012) 076501.

[3] Beenakker C. W. J., Annu. Rev. Condens. Matter Phys., 4 (2013) 113.

[4] Elliott S. R. and Franz M., Rev. Mod. Phys., 87 (2015) 137.

[5] Fu L. and Kane C. L., Phys. Rev. Lett., 100 (2008) 096407. 
[6] Fu L. and Kane C. L., Phys. Rev. B, 79 (2009) 161408.

[7] Oreg Y., Refael G. and von Oppen F., Phys. Rev. Lett., 105 (2010) 177002.

[8] Lutchyn R. M., Sau J. D. and Das Sarma S., Phys. Rev. Lett., 105 (2010) 077001.

[9] Stanescu T. D. and Tewari S., J. Phys.: Condens. Matter, 25 (2013) 233201.

[10] Nadj-Perge S., Drozdov I. K., Bernevig B. A. and Yazdani A., Phys. Rev. B, 88 (2013) 020407.

[11] Pientka F., Glazman L. I. and von Oppen F., Phys. Rev. B, 88 (2013) 155420.

[12] Klinovaja J., Stano P., Yazdani A. and Loss D., Phys. Rev. Lett., 111 (2013) 186805.

[13] Braunecker B. and Simon P., Phys. Rev. Lett., 111 (2013) 147202.

[14] Vazifeh M. M. and Franz M., Phys. Rev. Lett., 111 (2013) 206802.

[15] Mourik V., Zuo K., Frolov S. M., Plissard S. R., Bakkers E. P. A. M. and Kouwenhoven L. P., Science, 336 (2012) 1003.

[16] Das A., Ronen Y., Most Y., Oreg Y., Heiblum M. and Shtrikman H., Nature Phys., 8 (2012) 887.

[17] Deng M. T., Yu C. L., Huang G. Y., Larsson M., Caroff P. and Xu H. Q., Nano Lett., 12 (2012) 6414.

[18] Liu J., Potter A. C., Law K. T. and Lee P. A., Phys. Rev. Lett., 109 (2012) 267002.

[19] Bagrets D. and Altland A., Phys. Rev. Lett., 109 (2012) 227005.

[20] Pikulin D. I., Dahlhaus J. P., Wimmer M., SchomeRus H. and Beenakker C. W. J., New J. Phys., 14 (2012) 125011.

[21] Peng Y., Pientka F., Vinkler-Aviv Y., Glazman L. I. and von Oppen F., Phys. Rev. Lett., 115 (2015) 266804.

[22] Ben-Shach G., Haim A., Appelbaum I., Oreg Y., YaCOby A. and Halperin B. I., Phys. Rev. B, 91 (2015) 045403.

[23] de Juan F., Ilan R. and Bardarson J. H., Phys. Rev. Lett., 113 (2014) 107003.

[24] Liu D. E. and Baranger H. U., Phys. Rev. B, 84 (2011) 201308.

[25] Benjamin C. and Pachos J. K., Phys. Rev. B, 81 (2010) 085101.

[26] Law K. T., Lee P. A. and NG T. K., Phys. Rev. Lett., 103 (2009) 237001.

[27] Fidkowski L., Alicea J., Lindner N. H., Lutchyn R. M. and Fisher M. P. A., Phys. Rev. B, 85 (2012) 245121.

[28] Affleck I. and Giuliano D., J. Stat. Mech., 2013 (2013) P06011.

[29] Komijani Y. and Affleck I., Phys. Rev. B, 90 (2014) 115107.

[30] Clarke D. J., Alicea J. and Shtengel K., Nature Phys., 10 (2014) 877.

[31] Vasseur R., Dahlhaus J. P. and Moore J. E., Phys. Rev. X, 4 (2014) 041007.

[32] Lee Y.-W. and Lee Y.-L., Phys. Rev. B, 89 (2014) 125417.

[33] Chao S.-P., Schmidt T. L. and Chung C.-H., Phys. Rev. B, 91 (2015) 235125.

[34] Lutchyn R. M. and Skrabacz J. H., Phys. Rev. B, 88 (2013) 024511.
[35] Pikulin D. I., Komijani Y. and Affleck I., arXiv:1511.06319, (2015) .

[36] Zuo Z. W., Kang D. W, Wang Z. W. and Li L., arXiv:1603.01361, (2016).

[37] Gogolin A. O., Nersesyan A. A. and Tsvelik A. M., Bosonization and strongly correlated systems (Cambridge Univ. Press) 1998.

[38] Giamarchi T., Quantum Physics in One Dimension (Oxford Univ. Press) 2004.

[39] v. Klitzing K., Dorda G. and Pepper M., Phys. Rev. Lett., 45 (1980) 494.

[40] Tsui D. C., Stormer H. L. and Gossard A. C., Phys. Rev. Lett., 48 (1982) 1559.

[41] Laughlin R. B., Phys. Rev. Lett., 50 (1983) 1395.

[42] Wen X.-G., Int. J. Mod. Phys. B, 06 (1992) 1711.

[43] Wen X.-G., Adv. Phys., 44 (1995) 405.

[44] Halperin B. I., Phys. Rev. B, 25 (1982) 2185.

[45] BÉri B., Phys. Rev. Lett., 110 (2013) 216803.

[46] Altland A. and Egger R., Phys. Rev. Lett., 110 (2013) 196401.

[47] He J. J., Ng T. K., Lee P. A. and Law K. T., Phys. Rev. Lett., 112 (2014) 037001.

[48] Alicea J., Oreg Y., Refael G., Von Oppen F. and Fisher M. P., Nature Phys., 7 (2011) 412.

[49] Maslov D. L., Stone M., Goldbart P. M. and Loss D., Phys. Rev. B, 53 (1996) 1548.

[50] Winkelholz C., Fazio R., Hekking F. W. J. and SchÖN G., Phys. Rev. Lett., 77 (1996) 3200.

[51] Takane Y. and Koyama Y., J. Phys. Soc. Jpn., 66 (1997) 419.

[52] Affleck I., Caux J.-S. and Zagoskin A. M., Phys. Rev. B, 62 (2000) 1433.

[53] Titov M., Müller M. and Belzig W., Phys. Rev. Lett., 97 (2006) 237006.

[54] Fisher M. P. A., Phys. Rev. B, 49 (1994) 14550.

[55] Yoshioka D., The Quantum Hall Effect (Springer) 2002.

[56] Chang C.-Z., Zhang J., Feng X., Shen J., Zhang Z., Guo M., Li K., Ou Y., Wei P., Wang L.-L., Ji Z.Q., Feng Y., Ji S., Chen X., Jia J., Dai X., Fang Z., Zhang S.-C., He K., WANG Y., Lu L., MA X.-C. and Xue Q.-K., Science, 340 (2013) 167.

[57] Blonder G. E., Tinkham M. and Klapwijk T. M., Phys. Rev. B, 25 (1982) 4515. 